susceptibility to apoptosis, heightened permeability and enhanced endothelial proliferation. Substantial changes in bioenergetics of ECs, including higher rates of glycolysis, have been reported in PAH patients. However, the mechanisms underlying alterations in energy production have not been identified.

Methods We measured glycolysis in blood outgrowth endothelial cells (BOECs) from HPAH patients carrying mutations in BMPR2 and IPAH patients to confirm the metabolic abnormalities previously. We also employed an unbiased genome-wide microarray and proteomic screening approach to detect miRNAs and proteins dysregulated in the same groups to determine the mechanisms underlying abnormal endothelial glycolysis.

Results HPAH and IPAH BOECs recapitulated the metabolic phenotype previously observed in PAECs. These alterations were found to be associated with the downregulation of miR-124 and the upregulation of its known target, splicing factor polypyrimidine-tract-binding protein (PTBP1). We also demonstrated that increased PTBP1 promotes the switching in expression of two forms of pyruvate kinase, PKM1 and PKM2, resulting in an increase of aerobic glycolysis, consequently increasing cell proliferation (mechanism schematized in Figure 1). Overexpression of miR-124, or siRNA silencing of PTPB1, restoring normal expression levels of PKM2, also restored normal proliferation and glycolysis in HPAH BOECs. Finally, we observed reduced miR-124 and increased PTPB1 and PKM2 expression in a well-established rat model of $\mathrm{PAH}$, characterised by endothelial proliferation, supporting the presence of this mechanism in vivo.

Conclusions Loss of function of BMPR2 results in the downregulation of miR-124 and consequently in the glycolytic abnormalities reported in PAH ECs. Therefore, the manipulation of this miRNA, or its targets, could represent a novel and effective strategy to achieve clinical benefits in the treatment of PAH.

\section{S85 REDUCTION OF CD68 MACROPHAGES CAUSES GENDER SPECIFIC SPONTANEOUS PULMONARY ARTERIAL HYPERTENSION IN MICE}

A Zawia, N Arnold, A Braithwaite, J Pickworth, K Hopkinson, J Iremonger, G Miller, A Lawrie. The University of Sheffield, Sheffield, UK

\subsection{6/thoraxjnl-2016-209333.91}

Introduction Macrophages are proposed to play an important regulatory role in the pathogenesis of pulmonary arterial hypertension (PAH) as excessive infiltration detected around vascular lesions in patients and animal models. The exact 'causal' role for macrophages, and whether their presence or absence is required for the vascular remodelling seen in $\mathrm{PAH}$ remains unclear.

Objectives Using a novel inducible macrophage depletion model (MacLow mouse) we aimed to determine the role of macrophages in pulmonary arterial remodelling associated with PAH.

Methods Macrophage depletion was induced in MacLow mice by administration of doxycycline, where macrophage-specific induction of the cytotoxic diphtheria toxin A chain (DTA) is driven by the CD68 promoter. Mice were phenotyped for PAH by echocardiography, closed chest cardiac catheterization and immunohistochemistry (IHC) after 6 weeks. To investigate the origin of the effector cells, male chimeric mice were generated, and the disease stimulated by inducing macrophage ablation with doxycycline. Furthermore, to study gender-specificity of the disease phenotype, MacLow mixed gender chimeric mice were produced, and macrophage ablation induced as previous.
Results Interestingly male but not female MacLow mice developed a PAH phenotype compared to controls (RVSP of 66.1 $\mathrm{mmHg}$ vs $24.5 \mathrm{mmHg}, \mathrm{p}<0.0001, \mathrm{n}=5-8$ ), associated with increased right ventricular Hypertrophy (RVH 0.264 vs 0.226 , $\mathrm{p}<0.001, \mathrm{n}=8$ ) and pulmonary vascular remodelling. IHC analysis of diseased lungs demonstrated increased iNOS- |CD206 $+\mid \mathrm{F} 4 / 80+$ macrophages suggesting a M2-like macrophage population drive the PAH phenotype in these mice. The bone marrow transplant studies shows that bone marrow (BM) derived cells contribute in the development of the disease phenotype as wild type BM cells attenuate disease progression. Moreover, female BM transplanted into male mice alleviate but does not protect them from developing PAH.

Conclusion Development of PAH in male MacLow mice suggests that macrophages play a causal role in pulmonary vascular remodelling. Results suggest that the phenotype is driven by lung resident M2- like macrophages with a contribution from bone marrow derived cells. A study to examine the probable protective effect of Oestrogen is now underway to further investigate the implication of gender difference in the incidence of $\mathrm{PAH}$ in this model.

\section{S86 UNDERSTANDING THE ROLE OF GCN2 IN MODULATING BMP SIGNALING USING DROSOPHILA - IMPLICATIONS FOR PULMONARY HYPERTENSION}

E Malzer, CS Dominicus, SJ Marciniak. University of Cambridge, Cambridge, UK

\subsection{6/thoraxjn--2016-209333.92}

Introduction and objectives The majority of inherited cases of pulmonary arterial hypertension (PAH) are caused by mutations that affect the Bone Morphogenetic Protein (BMP) receptor 2. Recently, patients with pulmonary veno-occlusive disease (PVOD), a rare subtype of pulmonary hypertension, were found to have mutations of EIF2AK4. This gene encodes the kinase GCN2, which regulates cellular responses to amino acid availability by phosphorylating the translation initiation factor eIF2a. Phosphorylation of eIF2a reduces protein synthesis and induces expression of a transcription factor called ATF4. It is unclear if BMP and GCN2 signalling are functionally related and so we set out to test this using the model organism Drosophila melanogaster.

Methods We examined the development of wing veins in Drosophila because this is known to depend on BMP signalling. We manipulated the expression of dGCN2 and its antagonistic phosphatase dPPP1R15 by RNA interference. Morphology of the wing veins was examined in adult flies and early developmental signalling was measured in pupating animals. Similar experiments performed in animals with defective BMP signalling tested for a genetic interaction between eIF2a phosphorylation and BMP signalling.

Results We observed that depletion of dGCN2 in the developing wing by RNA interference enhanced BMP signalling as evidenced by hyperphosphorylation of MAD, the Drosophila orthologue of SMAD1. This was associated with development of ectopic wing veins in the regions of RNAi expression. In contrast, knock down of the eIF2a phosphatase dPPP1R15 selectively impaired anterior crossvein formation. The effects of dPPP1R15 depletion were cell-autonomous suggesting an effect on downstream BMP signalling rather than on ligand secretion. The vein phenotypes were highly sensitive to manipulation of BMP signalling. Both in insect and mammalian cells, ATF4 expression inhibited BMP signalling 
suggesting a mechanism for the crosstalk between BMP and GCN2.

Conclusion We have discovered in Drosophila that GCN2 activation modulates BMP signalling. This effect is mediated, at least in part, by the downstream transcription factor ATF4, which inhibits the phosphorylation of MAD (insect SMAD1). Our findings indicate that this pathway is conserved between insects and mammals and this model may shed light on the pathogenesis of $\mathrm{PAH}$ and PVOD.

\section{S87 DEFICIENCY OF TOLL-LIKE RECEPTOR 3 (TLR3) EXACERBATES PULMONARY HYPERTENSION IN MICE}

${ }^{1}$ AAR Thompson, ${ }^{1} \mathrm{ND}$ Arnold, ${ }^{1} \mathrm{AT}$ Braithwaite, ${ }^{1} \mathrm{HL}$ Casbolt, ${ }^{1} \mathrm{~J}$ Iremonger, ${ }^{1} \mathrm{JA}$ Pickworth, ${ }^{2} \mathrm{C}$ Monaco, ${ }^{2} \mathrm{JE}$ Cole, ${ }^{1}$ I Sabroe, ${ }^{1} \mathrm{~A}$ Lawrie. 'University of Sheffield, Sheffield, UK; ${ }^{2}$ University of Oxford, Oxford, UK

\subsection{6/thoraxjnl-2016-209333.93}

Introduction The mechanisms regulating aberrant vascular remodelling in pulmonary arterial hypertension $(\mathrm{PAH})$ are poorly understood and treatments targeted at halting or reversing this process are lacking. Toll-like receptor 3 (TLR3) is a viral sensor and more recently has been established as a sensor of endogenous damage signals, responding to mRNA released by damaged cells. TLR3 signalling induces pro- and anti-inflammatory cytokine production and regulates inflammation-associated apoptosis and tyrosine kinase signalling. In a model of systemic arterial injury, TLR3 signalling was shown to modulate neointimal remodelling in a protective manner. TLR3 is also expressed in pulmonary artery smooth muscle (PASMCs) and endothelial cells (PAECs). We therefore hypothesised that TLR3 would play roles in pulmonary vascular remodelling.

Methods TLR3-deficient (TLR3-/-) or wild-type C57BL/6 (WT) mice were exposed to hypoxia (10\% Oxygen) and given Sugen 5416 (weekly $20 \mathrm{mg} / \mathrm{kg}$ subcutaneous injections) or maintained in normoxic conditions for 3 weeks. Haemodynamic (cardiac catheterisation and echocardiography) and histological assessments were performed after 3 weeks. Human PASMCs were serum-starved before stimulation with PDGF or poly(I:C) and proliferation was assessed after 72 hours.
Results TLR3 - / - mice developed a markedly exaggerated phenotype of PAH in response to Sugen/Hypoxia with increased right ventricular systolic pressures (WT $51.6 \mathrm{mmHg} \pm 4.6$ vs. TLR3-/- $73.0 \mathrm{mmHg} \pm 6.8 ; \mathrm{p}<0.05$, mean \pm SEM, $\mathrm{n}=6$ ), increased muscularisation of small pulmonary arteries and reduced right ventricular cardiac output (WT 424.2 RVUmin$1 \pm 84.2$ vs. TLR $3-/-283.3$ RVUmin- $1 \pm 18.4$, mean \pm SEM, $\min \mathrm{n}=6$ ) after 3 weeks. Poly(I:C) suppressed PDGF-induced PASMC proliferation in a dose-dependent manner.

Conclusions We have shown that mice deficient in TLR3 develop a markedly exaggerated haemodynamic pulmonary hypertension phenotype and human PASMC proliferation is suppressed by the TLR3 ligand, poly(I:C). Together these data imply that TLR3 signalling in disease mediates a protective phenotype in keeping with that observed in systemic vascular remodelling, and identify a protective pathway potentially amenable to therapeutic targeting.

\section{Tuberculosis: From Screening to Side Effects}

\begin{tabular}{l|l}
\hline 588 & NEITHER UK TUBERCULOSIS INFECTION TESTING \\
GUIDELINE APPEARS COST-EFFECTIVE IN A \\
CONTEMPORARY HIV INFECTED POPULATION
\end{tabular}

${ }^{1} S$ Capocci, ${ }^{2} \mathrm{~J}$ Sewell, ${ }^{2} \mathrm{C}$ Smith, ${ }^{1} \mathrm{I}$ Cropley, 'S Bhagani, ${ }^{1} \mathrm{~A}$ Solamalai, ${ }^{2} \mathrm{~S}$ Morris, ${ }^{2}$ A Abubakar, ${ }^{1} \mathrm{MA}$ Johnson, ${ }^{1} \mathrm{MCl}$ Lipman. ${ }^{1}$ Royal Free London NHS Foundation Trust, London, UK; ${ }^{2}$ University College London, London, UK

\subsection{6/thoraxjnl-2016-209333.94}

UK guidelines advise testing for latent tuberculosis infection (LTBI) in people with known HIV. Both National Institute for Health and Care Excellence (NICE) 2011 and 2016, and British HIV Association (BHIVA) guidelines use targeted testing, in comparison to those from other countries, notably the United States. None of these have been compared for cost-effectiveness in a contemporary HIV population.

\begin{tabular}{|c|c|c|c|c|c|c|}
\hline Strategy & $\begin{array}{l}\text { Total cost of strategy per } 10,000 \\
\text { people living with HIV }\end{array}$ & $\begin{array}{l}\text { Cases TB } \\
\text { prevented } \\
\text { (discounted) }\end{array}$ & $\begin{array}{l}\text { QALYs gained compared to no } \\
\text { testing (discounted) }\end{array}$ & $\begin{array}{l}\text { Cost/case } \\
\text { averted }\end{array}$ & $\begin{array}{l}\text { Cost/QALY compared } \\
\text { to no testing }\end{array}$ & $\begin{array}{l}\text { Incremental cost/QALY } \\
\text { compared to last strategy }\end{array}$ \\
\hline BHIVA 2011 & $f 749,274$ & 2.28 & 1.28 & $f 21,371$ & $f 37,952$ & EXTENDED DOMINANCE \\
\hline TST in BA & $f 749,660$ & 3.9 & 2.09 & $£ 12,566$ & $£ 23,429$ & $£ 23,429$ \\
\hline TST in BA and MI & $f 761,797$ & 4.49 & 2.43 & $£ 13,614$ & $£ 25,218$ & EXTENDED DOMINANCE \\
\hline NICE 2011 & f788,037 & 1.11 & 0.63 & $f 78,429$ & $f 139,281$ & DOMINATED \\
\hline IGRA in BA & $f 812,048$ & 6.83 & 3.85 & $\mathrm{f} 16,314$ & $£ 28,971$ & EXTENDED DOMINANCE \\
\hline IGRA in BA and MI & f865,959 & 9.06 & 5.1 & $\mathrm{f} 18,250$ & $\mathrm{f} 32,410$ & EXTENDED DOMINANCE \\
\hline IGRA in all & $£ 1,056,702$ & 10.17 & 5.72 & $f 35,030$ & $f 62,209$ & EXTENDED DOMINANCE \\
\hline NICE 2016 & $\mathrm{f} 1,058,522$ & 10.17 & 5.72 & $\mathrm{f} 35,234$ & $\mathrm{f} 62,571$ & DOMINATED \\
\hline TST\&IGRA in all & $f 1,219,154$ & 10.99 & 5.88 & $f 47,166$ & f88,139 & EXTENDED DOMINANCE \\
\hline $\begin{array}{l}\text { TST\&IGRA\&CXR\&IS } \\
\text { in all }\end{array}$ & f1,999,789 & 20.58 & 10.44 & $\mathrm{f} 63,142$ & f124,393 & EXTENDED DOMINANCE \\
\hline
\end{tabular}

BA - Black African, BHIVA - British HIV Association, CXR - chest X ray, IGRA - Interferon-gamma release assay, IS - induced sputum, MI - middle [TB] incidence countries, NICE - National Institute of Health and Care Excellence, QALY - Quality adjusted life year, TB - tuberculosis (includes active disease and subclinical tuberculosis cases), TST - tuberculin skin test. 